\title{
PHYSICAL STUDIES OF CYCLOHEXANE DERIVATIVES
}

\author{
Y.N. MURThy ${ }^{a}$, M.V. Ramanaiah ${ }^{b}$ and V.R. Murthy ${ }^{c}$ \\ ${ }^{a}$ Department of Physics, S.S.B.N. College, Anantapur - 515 001, India \\ ${ }^{b}$ Dept. of Physics, S.D.G.S. College, Hindupur - 515 202, India \\ ${ }^{c}$ Dept. of Physics, S.K. University, Anantapur - 515 003, India
}

(Received July 7, 1999; revised version November 15, 1999)

\begin{abstract}
Mean molecular polarizabilities, polarizability anisotropies and order parameters of two homologous series of cyclohexane derivatives namely trans-4-alkyl cyclohexyl 4'-cyano-phenyl ester and trans-4-alkyl cyclohexyl $4^{\prime}$-n-pentyloxy phenyl ester are evaluated using molecular vibration approach. The variation of polarizability and polarizability anisotropy with a number of carbon atoms in the alkyl chain is studied. In addition, the dependence of order parameter on temperature is reported and discussed.
\end{abstract}

PACS numbers: $61.30 .-\mathrm{v}$

\section{Introduction}

It is an established fact that the physical behaviour of liquid crystals is remarkably subtle and is strongly influenced by the molecular structure and the contents of the mesogenic molecules. Many liquid crystalline compounds consist of two benzene rings in the central rigid core. But when the more polarizable benzene group is replaced by a much less polarizable cyclohexane, most of the physical properties like polarizability anisotropy, viscosity, dielectric anisotropy, transition temperature, etc. change considerably. In the series of cyclohexane derivatives, especially the 4-cyanophenyl and 4- $n$ alkyloxy phenyl esters are of interest because of the comparatively high clearing temperature in spite of low optical anisotropy and low viscosity. On replacing one benzene ring by the cyclohexane ring the conjugated region of the molecule is markedly shortened and therefore the polarizability anisotropy becomes relatively small [1].

In this paper, we present the results of molecular polarizabilities, polarizability anisotropies, and the order parameters for two series of cyclohexane derivatives. The study of calculated and experimental values of the results of polarizabilities provides information of conjugated region of molecular structure. The polarizability anisotropy of trans-4-alkyl cyclohexyl $4^{\prime}$-cyanophenyl ester is higher than the 
TABLE I

Structural formulae and transition temperatures of the two series of cyclohexane derivatives.

(I) trans-4-alkyl cyclohexyl 4'-cyanophenyl ester

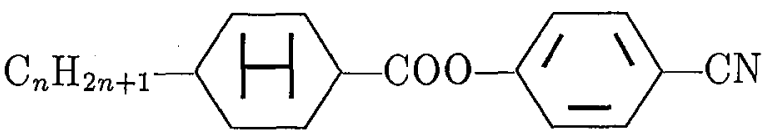

\begin{tabular}{c|c|c}
\hline$n$ & $T_{\mathrm{KN}}[\mathrm{K}]$ & $T_{\mathrm{NI}}[\mathrm{K}]$ \\
\hline 3 & 325.0 & 343.0 \\
4 & 327.7 & 342.9 \\
5 & 320.4 & 352.5 \\
\hline
\end{tabular}

(II) trans-4-alkyl cyclohexyl 4'-n-pentyloxy phenyl ester

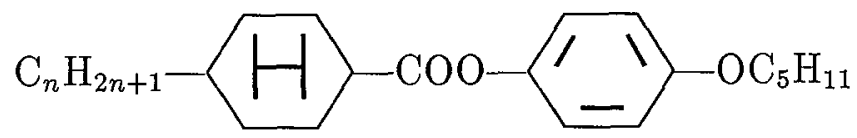

\begin{tabular}{c|c|c}
\hline$n$ & $T_{\mathrm{KN}}[\mathrm{K}]$ & $T_{\mathrm{NI}}[\mathrm{K}]$ \\
\hline 3 & 310.3 & 339.2 \\
4 & 302.5 & 339.5 \\
5 & 307.1 & 348.9
\end{tabular}

trans-4-alkyl cyclohexyl 4'-n-pentyloxy phenyl ester. This is due to the different substituent effects. If the alkyl chain (electron-repelling group $-\mathrm{OC}_{5} \mathrm{H}_{11}$ ) is substituted by the electron withdrawing group $(-\mathrm{CN})$, the polarizability anisotropy increases strongly [2].

The study of polarizabilities and their anisotropies is very important to understand the intermolecular interactions and also for the characterization of liquid crystalline substances. The packing fractions are important parameters in most of the molecular-statistical theories of the nematic state. It is interesting to note that the aromatic compounds possess remarkably higher values of the density than the cyclohexane derivatives. The density behaviour may be explained by the fact that the aromatic compounds contain relatively more heavy atoms (carbon) than the cyclohexane compounds with their higher contents of hydrogen. The packing fractions are a measure for the volume which is filled with material and therefore they are important parameters for molecular-statistical theories. Since large molecules possess higher mean polarizability values they often have higher packing fractions [3]. There are different theoretical models to evaluate mean molecular polarizabilities. Hauser et al. [4] have discussed the different internal field models. In all these models temperature dependence of refractive indices and densities are used to evaluate the mean molecular polarizabilities. But in our recent communications $[5,6]$ we have given a method namely "molecular vibration approach", in 
which the mean polarizabilities are evaluated using the vibrational frequency data. By employing the present method we have estimated the mean molecular polarizabilities and polarizability anisotropies. By applying Vuks' [7] internal field model order parameters are also evaluated for the two homologous series of cyclohexane derivatives.

The structural formulae and the transition temperatures of the two cyclohexane series namely

(I) trans-4-alkyl cyclohexyl 4'-cyanophenyl ester and

(II) trans-4-alkyl cyclohexyl 4'-n-pentyloxy phenyl ester are given in Table I.

\section{Theory \\ 2.1. Estimation of mean molecular polarizability}

The interaction of the electrons of the molecules with the incident electromagnetic radiation is usually treated by both classical and quantum concepts. But the validity of these two concepts is very much proved and both the treatments are considered. The originality of the present method of molecular vibration approach lies in correlating bond polarizabilities and stretching and bending force constants (molecular vibration parameters). The fact that bond polarizabilities, molecular vibration parameters, and related parameters like bond moment $(\mu)$ are used in explaining apparent molar Kerr constant of tetrahedrally symmetric molecules, gives the clue for a possible relationship between polarizability and molecular vibration parameters.

As the detailed theoretical procedure is given in our earlier communications $[5,6]$ we present here only the final relation which connect longitudinal $\left(b_{\mathrm{L}}\right)$ and transverse $\left(b_{\mathrm{T}}\right)$ bond polarizabilities with a mean amplitude of vibration $\left(\sigma^{1 / 2}\right)$

$$
b_{\mathrm{L}}+2 b_{\mathrm{T}}=C p^{j} j^{n r} \sigma^{1 / 2}
$$

where $C$ is a constant equal to $5.24 \times 10^{-15}, p$ is characteristic of the atom and is equal to $1,1.2,1.3,1.4$, and 1.5 accordingly as it belongs to $2^{\text {nd }}, 3^{\text {rd }}, 4^{\text {th }}$, and $5^{\text {th }}$ or $6^{\text {th }}$ row in the periodic table, $n$ is +1 or -1 accordingly as the bond is non-hydride or hydride and $r$ is the saturation factor.

Now the average polarizability $(\alpha)$ of the molecule is given by

$$
\alpha=\frac{1}{3} \sum n_{i}\left(b_{\mathrm{L}}+2 b_{\mathrm{T}}\right)_{i}
$$

where $n_{i}$ is the number of bonds of the type $i$.

\subsection{Estimation of molecular polarizability anisotropy}

Generally to estimate the anisotropy of polarizability $\left(a_{\|}-\alpha_{\perp}\right)$ from $\alpha_{e}$ and $\alpha_{0}$, the extrapolation procedure of Haller et al. [8] is used. In this method the value of $\log \left(\alpha_{e}-\alpha_{0}\right)$ is plotted against $\log \left(T_{\mathrm{NI}}-T\right)$ and when the straight line is extrapolated up to $\log T_{\mathrm{NI}}$ (where $T_{\mathrm{NI}}$ is the nematic-isotropic temperature), the limiting value of $\left(\alpha_{e}-\alpha_{0}\right)$ is assumed to correspond to the value of $\left(a_{\|}-\alpha_{\perp}\right)$ of the molecule. But the anisotropy values obtained by this method are found to have an error of around $10 \%$. So, in addition to this method, we have also 
used the procedure suggested by Prasad [9]. In this method the polarizability anisotropy is calculated from the value of $a_{\|}$. While evaluating $\alpha_{\|}$the molecular axis is taken along the line joining the centres of the benzene ring and cyclohexane ring on the basis of structural formula, assuming that the molecules have the all-trans-conformation. When a bond makes an angle $\theta$ with the molecular axis, the polarizability parallel to the molecular axis summed for all bonds is given by

$$
\alpha_{\|}=\sum \beta_{1} \cos ^{2} \theta+\sum \beta_{\mathrm{t}} \sin ^{2} \theta
$$

where $\beta_{1}$ and $\beta_{\mathrm{t}}$ are bond polarizabilities parallel and perpendicular to the bond, respectively.

\subsection{Estimation of order parameter}

The orientational order parameter $S$ is defined as [10]

$$
S=(1 / 2)\left\langle 3 \cos ^{2} \theta-1\right\rangle,
$$

where $\theta$ is the angle made by the long molecular axis with the preferred direction and the brackets denote an average over the molecules in a microscopic volume.

To calculate the polarizabilities of the molecules and the order parameter $(S)$ from birefringence data, Lorenz-Lorenz formula cannot be employed, because its validity is restricted to cases where there is a spherical (or cubic) symmetry in connection with the molecular arrangement. According to Vuks the ratio of the average local electric field to the applied field is the same for the two cases when the applied field is parallel and perpendicular to the optic axis of the medium.

In Vuks' model the order parameter is given by

$$
S=\frac{\bar{\alpha}}{\left(a_{\|}-\alpha_{\perp}\right)} \frac{\left(n_{e}^{2}-n_{0}^{2}\right)}{\left(n^{2}-1\right)}
$$

where $n^{2}=\left(n_{e}^{2}+2 n_{0}^{2}\right) / 3$ and $\bar{\alpha}$ is the mean polarizability estimated from molecular vibration approach. $a_{\|}$and $\alpha_{\perp}$ are the polarizabilities in a direction parallel and perpendicular to the optic axis, respectively. The necessary refractive indices are taken from the work of Takahashi et al. [11].

\section{Results and discussion}

The mean molecular polarizabilities estimated by the present method are presented in Table II for the two homologous series. The variation of molecular polarizability with a number of carbon atoms is shown in Fig. 1. The observation of polarizability data reveals that polarizabilities are almost independent of temperature. The values of $\alpha$ are higher for the pentyloxy phenyl ester than cyanophenyl ester. This can be understood from the fact that substitution of CN group in the place of alkyl chain decreases the polarizability. The values of the molecular polarizabilities computed in the present investigation agree well with the experimental values [8]. These values of polarizabilities provide a test for the additivity schemes and also yield information on the conformation of the terminal alkyl chain.

The polarizability anisotropies estimated by different methods in the two homologous series of cyclohexane derivatives are also presented in Table II. It is clear from the data that $\Delta \alpha$ is higher for a cyanophenyl ester compound. As it 


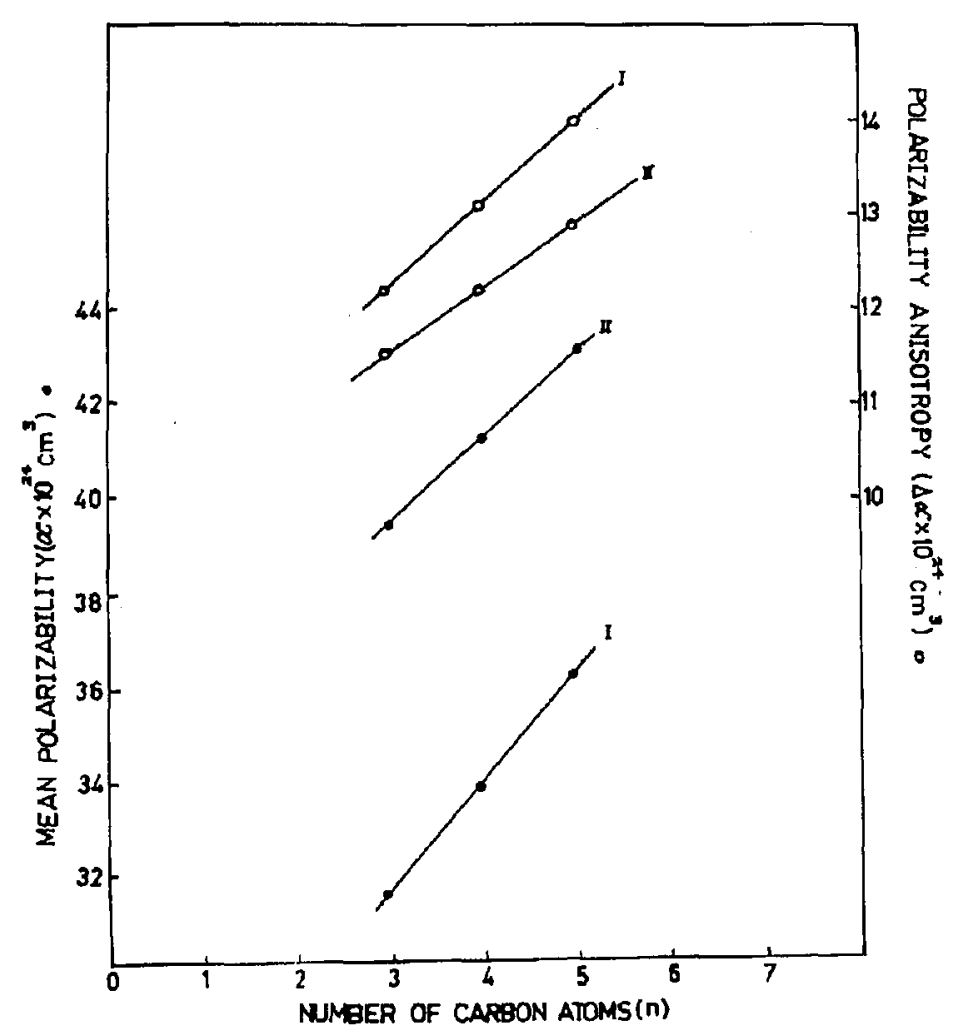

Fig. 1. Variation of molecular polarizability (filled circles) and polarizability anisotropy (open circles) with a number of carbon atoms in the series I \& II.

is already mentioned, the presence of the $\mathrm{CN}$ group in the end chain increases the polarizability anisotropy strongly. From the chemical studies it has long been known that the end chain plays a vital part in the stability of the mesophase. The transition temperature and a number of other properties like anisotropy, order parameter, and excess specific heats, etc. show a pronounced change. From the results of the polarizability anisotropy presented in Fig. 1, it is clear that $\Delta \alpha$ increases more slowly as compared to $\alpha$ and hence the anisotropy of polarizability normalized against the mean polarizability decreases linearly with the increase in the chain length. This is due to the fact that the polarizability anisotropy arises from the core contribution. The covalent bond electron in the alkyl chain contributes more to the average polarizability than the polarizability anisotropy. This indicates that the covalent bond electron contribution is almost uniform in parallel and perpendicular components. As a result $\Delta \alpha / \alpha$ decreases with the addition of $\mathrm{CH}_{2}$ group to the alkyl chain. The variation of order parameters with the reduced temperature $\left(T_{\mathrm{NI}}-T\right) / T_{\mathrm{NI}}$ is shown in Figs. 2 and 3 . The order parameters are estimated using Vuks (4) internal field model. In the two series under study we observe the decrease in the order parameter with an increase in temperature. In most of the liquid crystalline phases, the motion of parts of the 
TABLE II

Mean molecular polarizabilities, polarizability anisotropies of the two homologous series of cyclohexane compounds.

\begin{tabular}{c|c|c|c|c|c}
\hline \hline$n$ & $\begin{array}{c}\text { Mean molecular polarizability } \\
\left(\alpha \times 10^{24} \mathrm{~cm}^{3}\right)\end{array}$ & \multicolumn{3}{|}{$\begin{array}{c}\text { Polarizability anisotropy } \\
\left(\Delta \alpha \times 10^{24} \mathrm{~cm}^{3}\right)\end{array}$} \\
\hline & $\begin{array}{c}\text { Present } \\
\text { method }\end{array}$ & $\begin{array}{c}\text { Experimental } \\
\text { value [1] }\end{array}$ & $\begin{array}{c}\text { Present } \\
\text { method }\end{array}$ & $\begin{array}{c}\text { Experimental } \\
\text { value [11] }\end{array}$ & $\begin{array}{c}\text { Haller's } \\
\text { extrapolation }\end{array}$ \\
\hline \multicolumn{5}{c}{ trans-4-alkyl cyclohexyl 4'-cyanophenyl ester } \\
\hline 3 & 31.65 & 30.13 & 12.46 & 12.41 & 7.77 \\
4 & 34.04 & 32.44 & 13.20 & 13.46 & 9.21 \\
5 & 36.43 & 35.20 & 14.16 & 14.22 & 10.15 \\
\hline \multicolumn{5}{|c|}{ trans-4-alkyl cyclohexyl $4^{\prime}-n$-pentyloxy phenyl ester } \\
\hline 3 & 39.51 & 38.16 & 11.52 & 11.78 & 12.52 \\
4 & 41.33 & 40.62 & 12.0 & 12.05 & 12.95 \\
5 & 43.15 & 43.76 & 12.90 & 13.77 & 14.52
\end{tabular}

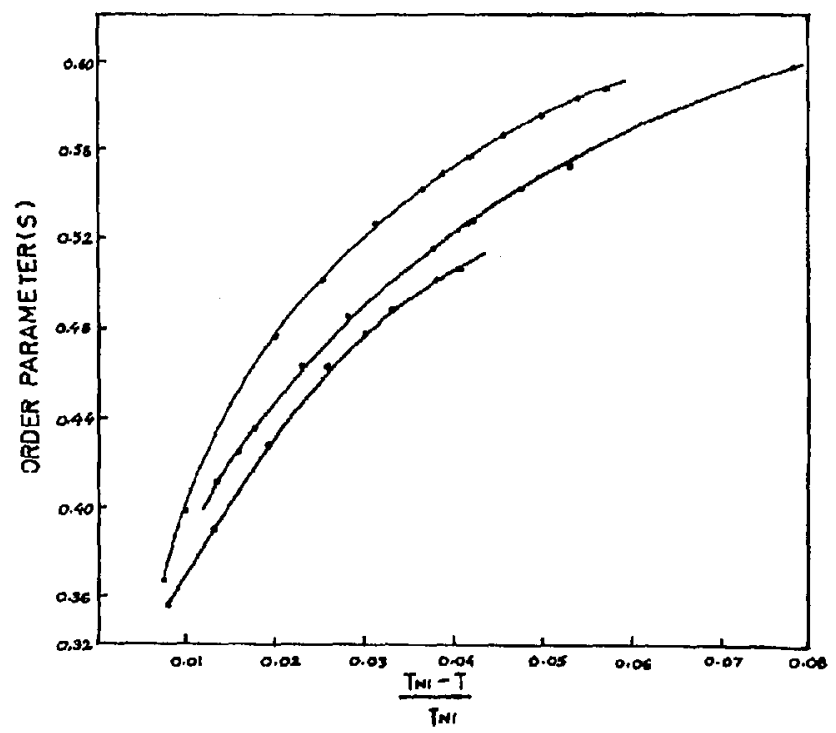

Fig. 2. Variation of the order parameter with the reduced temperature $\left(T_{\mathrm{NI}}-T\right) / T_{\mathrm{NI}}$ in series I (trans-4-alkyl cyclohexyl 4'-cyanophenyl ester).

molecule, e.g. free rotation about the single bonds (as in the case of $\mathrm{CH}_{2}$ groups at the end of the molecule), is always possible. The fractional number of molecules in which such motions take place and the amplitudes of such motions increase with increasing temperature. As a result there should be a small but systematic decrease in the optical anisotropy with increasing temperature and hence the order 


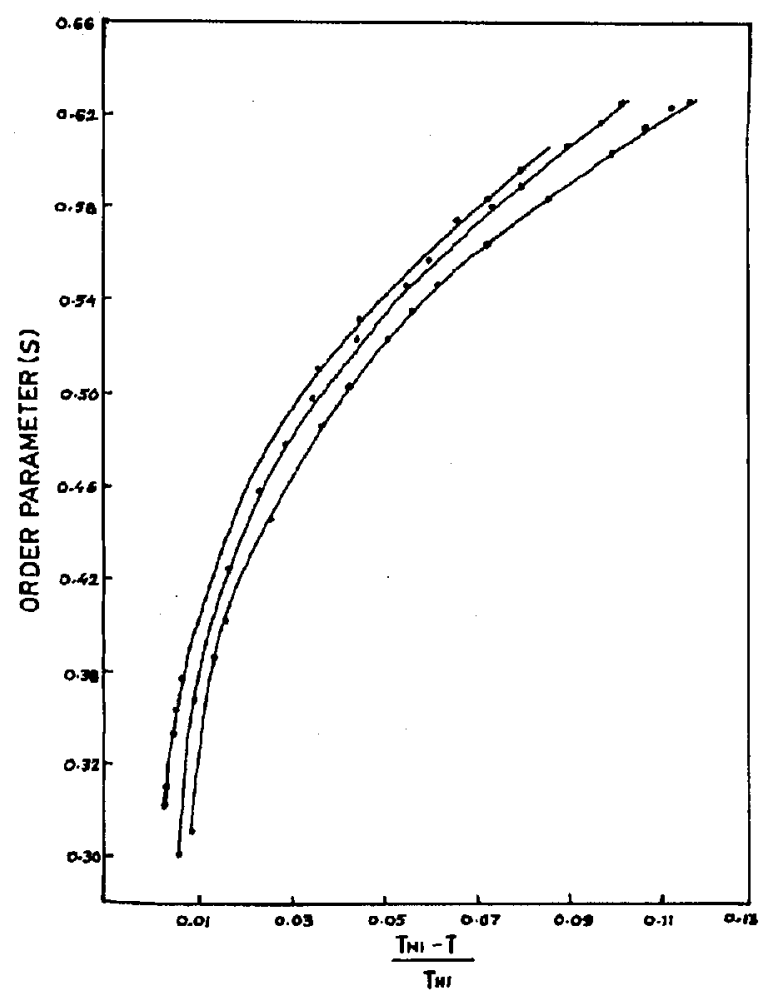

Fig. 3. Variation of the order parameter with the reduced temperature $\left(T_{\mathrm{NI}}-T\right) / T_{\mathrm{NI}}$ in series II (trans-4-alkyl cyclohexyl 4'-n-pentyloxy phenyl ester).

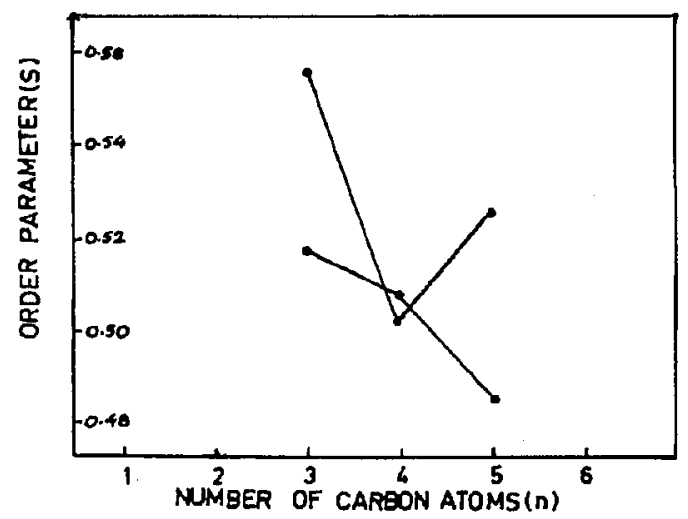

Fig. 4. Variation of the order parameter with the number of carbon atoms $(n)$ for the two series at the reduced temperature $\left(T_{\mathrm{NI}}-T\right) / T_{\mathrm{NI}}=0.04$.

parameter. The variation of order parameter with a number of carbon atoms in the alkyl chain at a given reduced temperature $\left(T_{\mathrm{NI}}-T\right) / T_{\mathrm{NI}}=0.040$ is studied in both the homologous series and presented in Fig. 4. The order parameters of series I 
are found to be much higher than those of series II. For materials with relatively high polarizability anisotropy, i.e. trans-4-alkyl cyclohexyl $4^{\prime}$-cyanophenyl ester, a relatively strong increase in $\alpha_{\perp}$ with increasing temperature is found. This leads to a higher order parameter. In the case of materials with a smaller polarizability anisotropy, for example trans-4-alkyl cyclohexyl-4'-n-pentyloxy phenyl ester the temperature change of $\alpha_{\perp}$ is smaller and hence this leads to a relatively low order parameter. In addition to this, we also find a distinct alternation of the order parameter in the first homologous series, which is often found for some properties of liquid crystal homologous series [12]. The alternation of the order parameter within the homologous series is obviously due to the alternating change of the molecular polarizability anisotropy caused by the alternation of the bonding angle in the aliphatic chain.

\section{Acknowledgments}

The authors are thankful to Prof. Pelzl, Martin-Luther Universität, Halle, Germany for sending his recent reprints. Also the authors greately acknowledge the help of Prof. Dr. Demus and Prof. Dr. Hauser, Germany in this work. The author (Y.N.M.) wish to record his deep sense of gratitude to sri. P.L.N. Reddy, Correspondent, S.S.B.N. College, Anantapur for his constant encouragement and moral support for the conduct of research activities in the institution.

\section{References}

[1] A. Hauser, G. Pelzl, C. Selbmann, D. Demus, Mol. Cryst. Liq. Cryst. 91, 97 (1983).

[2] H.S. Subramhanyam, C.S. Prabha, D. KrishnaMurti, Mol. Cryst. Liq. Cryst. 28, 201 (1974).

[3] Selected Topics in Liquid Crystal Research, Ed. H.-D. Koswig, Akademie-Verlag, Berlin 1990, p. 30.

[4] A. Hauser, D. Demus, Wiss.Z. Univ. Halle XXXVII, 137 (1988).

[5] Y.N. Murthy, V.R. Murthy, R.N.V. Ranga Reddy, Polish. J. Chem. 71, 460 (1997).

[6] Y.N. Murthy, V.R. Murthy, R.N.V. Ranga Reddy, Acta Phys. Pol. A 91, 1069 (1997).

[7] M.F. Vuks, Opt. Spectrosc. 20, 361 (1966).

[8] I. Haller, H.A. Huggins, H.R. Lilienthal, T.R. Meguire, J. Phys. Chem. 77, 950 (1973).

[9] S. Prasad, Acta Cienc. Indica 3, 244 (1977).

[10] S. Chandra Sekhar, Liquid Crystals, Cambridge University Press, New York 1977, p. 38.

[11] M. Takahashi, S. Mita, S. Kondo, Mol. Cryst. Liq. Cryst. 132, 53 (1986).

[12] A. Hauser, D. Demus, Z. Phys. Chem. Leipz. 270, 1057 (1989). 